\section{Orfandade por aids ou por homicídio apresenta efeitos sobre o estado nutricional das crianças?}

\section{Does orphanhood by aids or by homicide effect nutritional status of children?}

\section{Bruna Bronhara'}

Ivan Franca-Junior"

\section{Wolney Lisboa Conde ${ }^{\text {III }}$}

' Departamento de Epidemiologia da Faculdade de Saúde Pública da Universidade de São Paulo.

" Departamento de Saúde Materno Infantil da Faculdade de Saúde Pública da Universidade de São Paulo.

II' Departamento de Nutrição da Faculdade de Saúde Pública da Universidade de São Paulo.

Financiamento: Conselho Nacional de Desenvolvimento Científico e Tecnológico (Processo 476210/2004-6) e pela Fundação de Amparo à Pesquisa do Estado de São Paulo (Processos 2003/10883-5, 2005/59566-7, 06/50553 -2)

Correspondência: Bruna Bronhara. Departamento de Epidemiologia, Faculdade de Saúde Pública, Universidade de São Paulo. Av. Dr. Arnaldo, 715, CEP 01246-904 São Paulo, SP. E-mail: brunabronhara@usp.br

\section{Resumo}

O artigo tem como objetivos avaliar o estado nutricional de órfãos por aids ou homicídios residentes em São Paulo e estimar a associação de índices nutricionais com variáveis relacionadas à orfandade. Trata-se de estudo transversal de base domiciliar que utilizou amostra representativa de 484 indivíduos de 5 a 14 anos que perderam um ou ambos os pais durante os anos de 2000 e 2004 devido à aids ou a homicídio no município de São Paulo. A avaliação nutricional foi feita com o índice de massa corporal-para-idade (IMC) e da altura-para-idade (altura). A associação entre os índices nutricionais e as variáveis relacionadas à orfandade foi estimada em análise hierárquica, com uso de modelo de regressão linear múltiplo. Órfãos por aids ou homicídios diferiram quanto às características da orfandade e à idade média. As condições econômicas, domiciliares, o estado de saúde e o estado nutricional foram semelhantes entre os grupos. O déficit de IMC ocorreu em 1,3\% das crianças abaixo de 10 anos e em 2,1\% dos adolescentes. O déficit de altura ocorreu em $0,7 \%$ das crianças e em $4,0 \%$ dos adolescentes. O excesso de peso ocorreu em $19 \%$ e $20 \%$ das crianças e adolescentes, respectivamente. A análise hierárquica indicou ausência de efeito das variáveis relacionadas à orfandade sobre o IMC ou a altura; o principal determinante do estado nutricional foi de natureza econômica. Os órfãos por aids ou homicídio de São Paulo apresentaram estado nutricional semelhante e majoritariamente influenciado pela situação econômica. O perfil nutricional identificado no grupo, caracterizado pelo excesso de peso, sugere que os órfãos de São Paulo não apresentam riscos adicionais decorrentes da orfandade.

Palavras-chave: Crianças órfãs. Estado nutricional. Antropometria. Homicídio. Síndrome da Imunodeficiência Adquirida. 


\section{Abstract}

The aims of this paper were to evaluate the nutritional status of children orphaned by aids or homicides in the city of São Paulo and to estimate the association of nutritional indexes with orphanhood-related variables. The study was a household survey carried out between 2006 and 2007 . We sampled 484 children representative of São Paulo, 5-14 years old who lost either or both of their parents from aids or homicides between 2000 and 2004. We selected body-mass-index-for-age (BMI) and heightfor-age (height) as outcomes for analysis. Multiple linear regression in the light of a conceptual hierarchical approach was used for estimating BMI-for-age and height-forage associated factors. Children from aids and homicides groups differed in terms of orphanhood-related variables and age. Economic, household, health and nutritional conditions were similar between groups. Underweight accounted for $1.3 \%$ and $2.1 \%$ of children under the age of 10 and adolescents, respectively. Stunting accounted for $0.7 \%$ and $4.0 \%$ of children and adolescents, respectively. Overweight accounted for $19 \%$ and $20 \%$ of children and adolescents, respectively. BMI and height were unaffected by orphanhood-related variables after adjusting for selected classical determinants of nutritional status in the hierarchical model. Economic status was the main determinant of the nutritional profile. Nutritional status of children orphaned by aids or homicides in São Paulo was similar and mainly influenced by economic status. The nutritional profile, characterized by being overweight, suggests that these orphans have not shown additional risks due to those orphanhoodrelated variables.

Keywords: Child. Orphaned. Nutritional status. Anthropometry. Homicide. Acquired Immunodeficiency Syndrome.

\section{Introdução}

A saúde e a nutrição das crianças que perderam um ou ambos os pais podem ser afetadas em virtude do impacto econômico causado pela perda do adulto economicamente ativo ${ }^{1-4}$, redução dos cuidados dispensados à criança pela perda do cuidador primário ${ }^{3,5}$, maior exposição à discriminação, inclusive da qualidade alimentar, na nova família ${ }^{2}$, traumas psicológicos ${ }^{6}$, entre outros. Há ainda a possibilidade de que o estigma e a discriminação associados ao HIV/aids possam ampliar os efeitos negativos sobre a saúde da criança, por isolá-la do convívio social ou pela falta de acesso a serviços de saúde no momento que ela mais precisa de cuidados e apoio ${ }^{3}$.

Os estudos sobre orfandade e suas consequências para a saúde e estado nutricional de crianças foram conduzidos, em sua maioria, em países africanos, muitos dos quais envolvidos em conflitos armados ${ }^{7,8}$ ou com elevada prevalência de HIV/aids na população $0^{2,5,7-10}$.

A análise dos efeitos das condições advindas da orfandade* sobre o estado nutricional infantil usualmente toma como variável dependente os índices peso-para-altura, altura-para-idade, peso-para-idade e, com menor frequência, índice de massa corporal, dobras cutâneas ou circunferências corporais. A comparação entre os resultados obtidos nos principais estudos, contudo, aponta certa variabilidade em relação à magnitude e à direção destes efeitos ${ }^{5,6,8,10,11}$. Parte dessa variabilidade pode ser atribuída a fatores contextuais que atuam atenuando ou acentuando os efeitos da orfandade, e parte às propriedades dos índices nutricionais para capturar e conservar, biologicamente, estes efeitos.

Não foram localizados estudos nacionais ou internacionais com análises sobre as condições nutricionais dos órfãos brasileiros. É evidente que a literatura sobre órfãos se concentra na África subsaariana 
em função da elevada taxa de mortalidade de adultos influenciada pela epidemia de aids na região ${ }^{9}$. No Brasil, o estudo das condições nutricionais dessas crianças deve se centrar não somente no impacto da mortalidade por aids, mas também no impacto da mortalidade associada às elevadas taxas de homicídios. O quadro social dos homicídios no Brasil se caracteriza por acometer jovens, ou seja, indivíduos em idade reprodutiva, e em situação econômica desfavorável, fatores que podem formar um potencial agravante ao estado nutricional das crianças ${ }^{12}$.

Este trabalho avaliou o estado nutricional de órfãos por aids ou homicídios e sua associação com variáveis relacionadas à orfandade.

\section{Métodos}

Estudo transversal de base domiciliar que utilizou amostra probabilística de órfãos entre 5 e 14 anos completos de idade. Estas crianças haviam perdido um ou ambos os pais entre os anos de 2000 e 2004 por aids ou homicídio.

A seleção amostral foi realizada por conglomerados com tamanhos diferentes, realizada a partir das informações extraídas do cadastro do Programa de Aprimoramento das Informações sobre Mortalidade do Município de São Paulo (PROAIM), tais como nome completo, sexo, idade e endereço de todos os óbitos por aids e homicídio ocorridos entre 2000 e 2004. Os falecidos com menos de 18 anos, com endereços localizados fora da cidade de São Paulo ou endereços inválidos foram excluídos. Do total de casos elegíveis, 22.637 registros eram de homicídios e 5.977 de aids.

A amostra total de óbitos foi estimada em 4.042 registros, sendo $50 \%$ destinada aos óbitos por aids e a outra parte para óbitos por homicídio. O cálculo do tamanho amostral se baseou na expectativa de $5 \%$ de institucionalização para órfãos e nas expectativas de perdas nas fases de rastreamento e entrevista domiciliar. Os valores para estas estimativas foram extraídos da pesquisa de base domiciliar realizada em Porto Alegre com órfãos devido à aids ${ }^{13}$.

A amostragem foi realizada em estágios múltiplos e envolveu processos de estratificação e sorteio de conglomerados. Para delimitar a unidade primária de amostragem (UPA) foram utilizados os quatro primeiros dígitos do CEP.

Sinteticamente, o desenho da amostra dos registros de óbitos por homicídio foi composto por quatro estratos: dois formados por duas UPAs autorrepresentativas (UPAs grandes que não necessitaram de sorteio amostral), um formado pelas demais UPAs e um formado pelos endereços sem CEP. A amostra final foi equiprobabilística e totalizou 2.138 endereços. No caso da amostra dos registros de óbitos por aids, o desenho foi composto por dois estratos, o primeiro formado por UPAs com CEP e o segundo pelos endereços sem CEP. A amostra final foi equiprobabilística e composta por 2.081 endereços.

No caso dos óbitos por homicídios, foram sorteados um a cada dois órfãos para a avaliação antropométrica. No caso dos óbitos por aids este critério não foi aplicado em função do grupo reduzido de órfãos localizado durante o campo.

Os detalhes da etapa de rastreamento, bem como as principais fontes de perdas amostrais ocorridas neste processo, estão descritas na Figura 1. A amostra final totalizou 269 órfãos por aids e 215 órfãos por homicídio. A coleta dos dados ocorreu de setembro de 2006 a março de 2007.

A amostra utilizada no trabalho foi ponderada para a ausência de resposta, para que a representatividade do estudo não fosse afetada. Os pesos foram calculados por meio do modelo log-linear de Poisson, para o ajuste da distribuição dos respondentes à população total do estudo (peso de não resposta). Maiores detalhes encontram-se em Barros et al. ${ }^{14}$.

A variável dependente, estado nutricional, foi estimada com uso das medidas de peso e altura, coletadas conforme os procedimentos recomendados por Lohman et al. ${ }^{15}$. Cada medida foi realizada duas vezes 


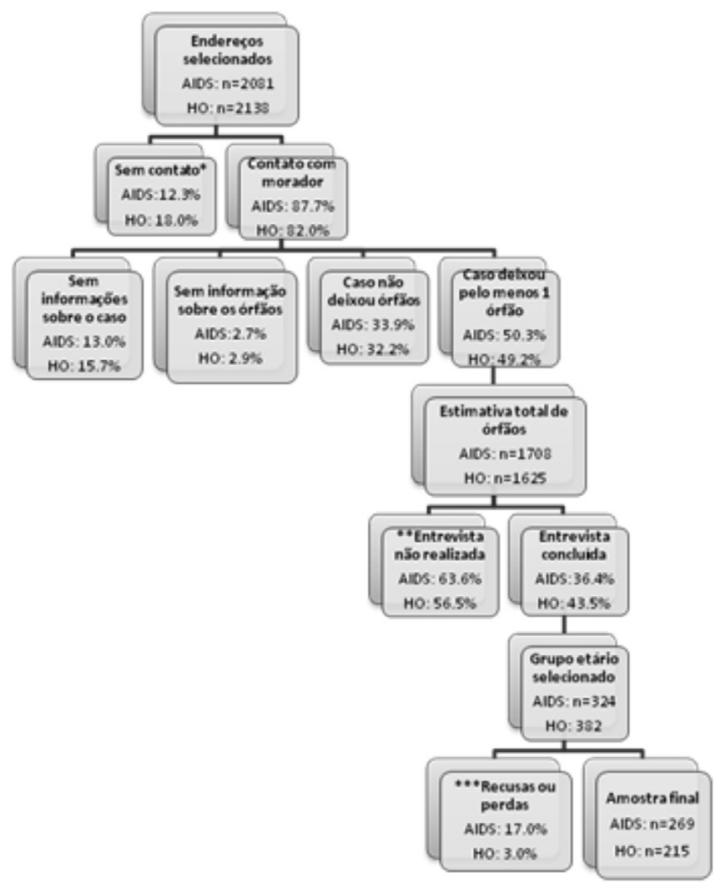

$\mathrm{HO}=$ homicídios $/ \mathrm{HO}=$ homicides

*Moradias destruídas, endereço inexistente ou ausência de moradores após três visitas domiciliares. / *House destroyed, non-existing address or no dwellers after three visits to the house.

** Mudança de domicílio, ausência de informação sobre o novo endereço do órfão, recusas em realizar entrevistas socioeconômicas, prisão do órfão ou falta de segurança em permanecer no local selecionado.

** Change of address, no information on the orphan's new address, refusal to answer socioeconomic interview, orphan incarcerated or lack of security to remain at the site selected.

*** Recusas, perdas ou institucionalização. / *** Refusals, losses or institutionalization.

Figura 1 - Fluxograma das perdas amostrais durante o rastreamento dos órfãos por aids e homicídio. São Paulo, Brasil, 20006-07.

Figure 1 - Flowchart of sample loss during the screening of children orphaned by aids or by homicides. São Paulo, Brasil, 2006-07.

seguidas e tomou-se a média dos valores. $\mathrm{O}$ índice de massa corporal foi calculado como: peso/altura ${ }^{2}$. O estado nutricional foi classificado em desnutrição e excesso de peso segundo os valores críticos do IMC por idade e sexo propostos por Conde e Monteiro ${ }^{16}$.

A adoção do IMC-para-idade (IMC) foi justificada por se tratar de uma população de 5 a 14 anos, diferentemente dos demais estudos identificados com órfãos, que abrangeram crianças até cinco anos de idade. Nesses casos, o uso de índices tradicionais como peso/altura e peso/idade mostraram-se adequados ${ }^{17}$, pois usualmente a taxa de crescimento das crianças é semelhante à taxa de ganho de peso. Entre crianças mais velhas e no início da adolescência a taxa de ganho de peso torna-se mais veloz que a taxa de crescimento, indicando a necessidade de um índice que controle a altura de maneira mais adequada, do tipo peso/altura ${ }^{\text {p }}$. No caso do IMC, $p$ vale 2. Além disso, há a necessidade de que o IMC seja também controlado pela idade, de modo que se tenha um índice não correlacionado com a altura para crianças de cada faixa etária. Atualmente, esse índice é utilizado na infância, entre crianças maiores e na adolescência ${ }^{8-19}$.

Os valores da altura foram convertidos em desvios padronizados (escore Z) poridade e sexo, segundo a referênciaWHO-200720. Valores do escore de altura-para-idade (altura) abaixo de -2 foram considerados déficit de crescimento ${ }^{17}$.

As variáveis independentes selecionadas foram: idade do órfão, sexo e cor da pele do órfão, renda familiar mensal per capita, 
escore econômico (detalhes no parágrafo seguinte), declaração da condição econômica da família após o óbito, grupo de idade do cuidador, sexo do cuidador, escolaridade do cuidador, residência em casa de alvenaria, coleta de lixo domiciliar, presença de água canalizada, estado de saúde atual do órfão segundo a percepção do cuidador, internações realizadas nos últimos 12 meses, tipo de orfandade, tempo de orfandade, idade em que a criança/adolescente ficou órfão, principal cuidador e causa da orfandade (aids ou homicídio).

Optou-se pela Classificação Econômica Brasil - 2008 (CCEB-2008)**, que utiliza informações sobre bens materiais (televisão, rádio, geladeira, entre outros) e escolaridade do chefe do domicílio, para criar uma pontuação e assim classificar os domicílios em classes econômicas. No entanto, a ausência de informações sobre freezer e escolaridade do chefe da família, na base de dados do presente estudo, impôs a necessidade de se adaptar esta classificação. Assim, calculou-se a variável escore econômico, a qual continha a pontuação obtida de acordo com os bens materiais do domicílio (exceto freezer). O escore econômico máximo possível de ser obtido no presente estudo, utilizando-se a pontuação do CCEB-2008, foi de 36 pontos.

Imputaram-se 30 casos faltantes da variável renda familiar a partir da regressão linear simples entre os casos existentes de renda familiar e escore econômico.

Na primeira parte da análise foram investigadas as diferenças demográficas, socioeconômicas, de saúde e de estado nutricional entre os grupos de órfãos por homicídio ou por aids.

Para estimar os fatores associados aos escores Z do IMC e altura, as variáveis foram organizadas em blocos hierárquicos, de acordo com o modelo conceitual do estado nutricional de crianças, adaptados do UNICEF $^{21}$. Em cada bloco hierárquico realizou-se análise de regressão linear múltipla para selecionar as variáveis associadas aos desfechos de interesse ao nível de $20 \%$.
As variáveis pré-selecionadas do bloco distal foram incluídas nos blocos inferiores. Consideraram-se fator de risco aquelas com nível de significância de 5\%. A descrição dos blocos de cada variável dependente encontra-se a seguir.

O IMC representa o balanço entre as necessidades e a ingestão total energética, as quais são influenciadas por fatores mais complexos e inter-relacionados, como estabilidade política e situação econômica, ou por fatores imediatos como a presença de doenças ou a ingestão de alimentos ${ }^{21}$. $\mathrm{O}$ desequilíbrio entre a necessidade e a ingestão energética influencia diretamente $\mathrm{e}$ em curto prazo os componentes corporais e, portanto, a massa corporal ${ }^{22}$. Assim, para o IMC, foram selecionadas as informações relacionadas ao estado nutricional atual do órfão. O bloco distal dos determinantes foi composto pelas informações socioeconômicas (renda familiar per capita atual) e biológicas (sexo e idade). O bloco de determinantes intermediários reuniu informações relacionadas aos cuidados dispensados à criança/adolescente, representadas pelas variáveis relacionadas à orfandade: tipo de orfandade, tempo da orfandade, causa da orfandade, idade em que ficou órfão e condição econômica após o óbito do falecido. Como hipótese, as crianças órfãs de ambos os pais, de idade mais nova ou que haviam ficado órfãs há pouco tempo poderiam apresentar os efeitos da orfandade de forma mais evidente do que as demais crianças. Neste bloco incluiu-se também a escolaridade do cuidador. Devido à ausência de informações sobre os padrões alimentares e atividade física dos órfãos, no bloco imediato inseriram-se somente as informações sobre o estado de saúde atual e internação no ano anterior à entrevista domiciliar.

Para a altura, selecionaram-se as informações que melhor sintetizassem os eventos passados do órfão, considerando que o da altura permite a leitura retrospectiva do estado nutricional. Neste caso, o bloco distal foi composto pelas informações biológicas e

\footnotetext{
** Disponível em http://www.abep.org/novo/CMS/Utils/FileGenerate.ashx? Id = 22.
} 
pelo escore econômico, ou seja, pelo patrimônio acumulado pela família até o momento da entrevista. Considerando as crianças que se tornaram órfãs em idade mais nova (nas quais o crescimento é mais acelerado) ou estavam órfãs há mais tempo (sofrendo continuamente as consequências negativas da orfandade até restringir o crescimento normal), o bloco intermediário foi composto também pelas variáveis relacionadas à orfandade e pelas informações sobre a condição econômica após o óbito. O bloco imediato de variáveis sobre a saúde também foi excluído por não possibilitar a análise temporal com o desfecho de interesse.

Foi investigada também a interação entre escore econômico ou renda familiar per capita, e a variável alteração da condição econômica da família após o óbito.

As análises estatísticas foram realizadas com uso do comando Survey do software Stata, o qual analisa os dados levando em conta o delineamento complexo da amostra. As estimativas foram apresentadas em valores de média e erro padrão (EP).

$\mathrm{O}$ protocolo de pesquisa foi aprovado pelo Comitê de Ética da Faculdade de Saúde Pública da Universidade de São Paulo (Of. COEP/26/2008 - Protocolo 1734).

\section{Resultados}

Os aspectos demográficos, socioeconômicos e domiciliares dos órfãos e seus cuidadores, segundo a causa da orfandade, estão descritos na Tabela 1. Os órfãos por aids eram mais velhos que os órfãos por homicídio e cuidados principalmente por pessoas de maior idade. A maior parte dos cuidadores era do sexo feminino, principalmente entre órfãos por homicídio.

Em média, os cuidadores apresentaram 6, 7 (EP: 0,3) anos de escolaridade. Os cuidadores de órfãos por homicídio tinham um ano de estudo a mais que os cuidadores de órfãos por aids. A média do escore econômico foi de 9,2 (EP: 0,2) pontos, que correspondeu a $25 \%$ do total possível de ser obtido.

Grande parte dos órfãos, de ambos os grupos, concentrou-se nas categorias inferiores de salário mínimo e escore econômico. A frequência de famílias morando em casa de alvenaria, com coleta de lixo regular e canalização da água foi praticamente universal (Tabela 1).

Os aspectos relacionados à orfandade estão descritos na Tabela 2. A orfandade paterna foi mais frequente entre órfãos por homicídio. A orfandade por homicídio ocorreu em idade mais precoce do que aquela observada nos órfãos por aids, o que resultou em maior tempo de orfandade entre órfãos por homicídio. O cuidado pela mãe foi mais frequente em órfãos por homicídio.

A piora na condição econômica da família em função do óbito de um ou ambos os pais foi significativamente maior para os órfãos por homicídio.

A Tabela 3 apresenta as frequências dos indicadores de saúde e de estado nutricional. A frequência de estado de saúde inadequado, de ocorrência de internações e de déficit de altura ou peso foi baixa em ambos os grupos. A frequência do excesso de peso foi aproximadamente 10 vezes a frequência do déficit de peso tanto entre órfãos por aids quanto entre órfãos por homicídio.

$O$ déficit de IMC ocorreu em $1,2 \%$ das crianças abaixo de 10 anos e em 2,1\% dos adolescentes. $\mathrm{O}$ déficit de altura ocorreu em $0,7 \%$ das crianças e em $4,0 \%$ dos adolescentes. O excesso de peso ocorreu em 19\% e $20 \%$ das crianças e adolescentes, respectivamente. Nenhuma destas características diferiu segundo o sexo.

Os efeitos brutos e ajustados dos determinantes biológicos, econômicos e da orfandade selecionados para os modelos múltiplos do IMC e da altura estão descritos na Tabela 4.

No modelo do IMC, selecionaram-se para a análise múltipla o bloco distal e a variável tipo de orfandade do bloco intermediário, a qual se associou ao IMC após o controle simultâneo para as demais variáveis deste nível. Nenhuma das variáveis imediatas utilizadas associou-se ao IMC. O modelo múltiplo final indicou que IMC associou-se ao salário mínimo, após ajuste para idade, sexo e tipo da orfandade. O 
Tabela 1 - Crianças órfãs por aids ou homicídio, de 5 a 14 anos, segundo características demográficas, socioeconômicas e domiciliares (\%). São Paulo, Brazil, 2006-07.

Table 1 - Children orphaned by aids or by homicide, aged 5-14 years, according to demographic, socioeconomic and household characteristics (\%). São Paulo, Brazil, 2006-7.

\begin{tabular}{|c|c|c|c|c|c|}
\hline \multirow[b]{2}{*}{ Características } & \multirow[b]{2}{*}{ Categorias } & \multirow[b]{2}{*}{$\begin{array}{c}\text { Total } \\
n=484 \\
(\%)\end{array}$} & \multicolumn{2}{|c|}{ Causa da orfandade } & \multirow[b]{2}{*}{$p$} \\
\hline & & & $\begin{array}{c}\text { aids } \\
n=269 \\
(\%)\end{array}$ & $\begin{array}{c}\text { Homicídio } \\
n=215 \\
(\%)\end{array}$ & \\
\hline \multicolumn{6}{|l|}{ Demográficas - órfão } \\
\hline \multirow[t]{2}{*}{ Grupo etário } & 5 a 9 anos & 55.1 & 40.6 & 50.6 & 0.00 \\
\hline & 10 a 14 anos & 44.9 & 59.4 & 39.4 & \\
\hline \multirow[t]{2}{*}{ Sexo } & Masculino & 47.0 & 47.1 & 47.0 & 0.97 \\
\hline & Feminino & 53.0 & 52.9 & 53.0 & \\
\hline \multirow[t]{4}{*}{ Cor de pele } & Branca & 37.0 & 42.7 & 34.9 & 0.36 \\
\hline & Preta & 8.1 & 10.4 & 7.3 & \\
\hline & Amarela & 0.4 & 0.0 & 0.5 & \\
\hline & Parda + Indígena* & 54.5 & 46.9 & 57.3 & \\
\hline \multicolumn{6}{|l|}{ Demográficas - cuidador } \\
\hline \multirow[t]{3}{*}{ Grupo etário } & 17 a 24 anos & 7.5 & 3.9 & 8.8 & 0.01 \\
\hline & 25 a 60 anos & 84.1 & 79.6 & 85.8 & \\
\hline & 61 anos ou mais & 8.4 & 16.5 & 5.4 & \\
\hline \multirow[t]{2}{*}{ Sexo } & Masculino & 4.5 & 9.0 & 2.7 & 0.03 \\
\hline & Feminino & 95.5 & 91.0 & 97.3 & \\
\hline \multicolumn{6}{|l|}{ Socioeconômicas } \\
\hline \multirow[t]{5}{*}{ Anos de estudo do cuidador } & Nenhum & 7.3 & 6.1 & 7.7 & 0.04 \\
\hline & $1 \mid--3$ anos & 12.0 & 16.1 & 10.4 & \\
\hline & $4 \mid--7$ anos & 33.4 & 42.0 & 30.1 & \\
\hline & $8 \mid--11$ anos & 43.5 & 31.1 & 48.3 & \\
\hline & 12 anos ou mais & 3.9 & 4.6 & 3.6 & \\
\hline \multirow[t]{5}{*}{ Salário mínimo per capita** } & $0 \mid-1 / 4$ & 28.4 & 25.9 & 29.3 & 0.62 \\
\hline & $1 / 4 \mid--1 / 2$ & 33.1 & 32.6 & 33.2 & \\
\hline & $1 / 2 \mid--1$ & 27.4 & 27.3 & 27.4 & \\
\hline & $1 \mid--2$ & 9.2 & 10.7 & 8.6 & \\
\hline & $>=2$ & 2.0 & 3.5 & 1.4 & \\
\hline \multirow[t]{4}{*}{ Escore econômico*** } & 3 a 6 pontos & 12.1 & 14.3 & 11.2 & 0.70 \\
\hline & 7 a 10 pontos & 59.7 & 57.8 & 60.5 & \\
\hline & 11a 14 pontos & 26.1 & 24.7 & 26.6 & \\
\hline & 15 a 18 pontos & 2.2 & 3.3 & 1.7 & \\
\hline \multicolumn{6}{|l|}{ Domiciliares } \\
\hline Vive em casa de alvenaria? & Sim & 91.7 & 91.6 & 91.7 & 0.97 \\
\hline Há coleta de lixo domiciliar? & Sim & 99.6 & 100.0 & 99.5 & 0.54 \\
\hline A água é canalizada? & Sim & 99.6 & 99.7 & 99.5 & 0.80 \\
\hline
\end{tabular}

* A categoria indígena apresentou apenas 4 casos; assim, foi inserida na categoria parda.

* The category indigenous had 4 cases; thus it was added to the brown category.

** Calculado de acordo com o salário mínimo durante o período da coleta de dados (R\$350,00).

** Calculated according to the minimum wage during the data collection period ( $R \$ 350.00$ ).

*** Escore econômico calculado segundo as ponderações dos critérios de Classificação Econômica Brasileira de 2008. Disponível em http://www. abep.org/novo/CMS/Utils/FileGenerate.ashx?id=22

*** Economic score calculated according to weights of the 2008 Brazilian Economic Classification criteria. Available at http://www.abep.org/novo/CMS/

Utils/FileGenerate. $a s h x ? i d=22$ 
Tabela 2 - Crianças órfãs por aids ou homicídio, de 5 a 14 anos, segundo características relacionadas à orfandade (\%). São Paulo, Brasil, 2006-07.

Table 2 - Children orphaned by aids or by homicide, aged 5-14 years, according to orphanhood-related variables (\%). São Paulo, Brazil, 2006-07.

\begin{tabular}{|c|c|c|c|c|c|}
\hline \multirow[b]{2}{*}{ Características } & \multirow[b]{2}{*}{ Categorias } & \multirow[b]{2}{*}{$\begin{array}{c}\text { Total } \\
n=484 \\
(\%)\end{array}$} & \multicolumn{2}{|c|}{ Causa da orfandade } & \multirow[b]{2}{*}{$p$} \\
\hline & & & $\begin{array}{c}\text { aids } \\
n=269 \\
(\%)\end{array}$ & $\begin{array}{c}\text { Homicídio } \\
n=215 \\
(\%)\end{array}$ & \\
\hline \multirow[t]{3}{*}{ Tipo de orfandade } & Paterna & 75.8 & 50.5 & 85.4 & 0.00 \\
\hline & Materna & 15.7 & 28.5 & 10.8 & \\
\hline & Ambos os pais & 8.5 & 21.0 & 3.8 & \\
\hline \multirow[t]{3}{*}{ Tempo da orfandade } & $1-3$ anos & 28.8 & 42.2 & 23.8 & 0.00 \\
\hline & 3-5 anos & 31.8 & 31.5 & 31.9 & \\
\hline & 5-7 anos & 39.4 & 26.3 & 44.3 & \\
\hline \multirow[t]{3}{*}{ Idade na orfandade } & $0-2$ anos & 28.6 & 16.4 & 33.2 & 0.00 \\
\hline & 3-5 anos & 34.2 & 27.6 & 36.7 & \\
\hline & 6-13 anos & 37.2 & 56.0 & 30.1 & \\
\hline \multirow[t]{4}{*}{ Principal cuidador } & Mãe & 60.2 & 43.3 & 66.5 & 0.00 \\
\hline & Avó materna & 7.7 & 11.1 & 6.5 & \\
\hline & Tios maternos & 15.2 & 17.6 & 14.2 & \\
\hline & Outros parentes & 16.9 & 28.0 & 12.8 & \\
\hline Piora da condição econômica após o óbito & Sim & 44.5 & 36.5 & 47.5 & 0.00 \\
\hline
\end{tabular}

Tabela 3 - Indicadores de saúde e de estado nutricional (\%) de crianças órfãs por aids e homicídio, de 5 a 14 anos de idade. São Paulo, Brasil, 2006-07.

Table 3 - Nutritional status and health indicators (\%) of children orphaned by aids or by homicide aged 5-14 years. São Paulo, Brazil, 2006-07.

\begin{tabular}{|c|c|c|c|c|c|}
\hline \multirow[b]{2}{*}{ Características } & \multirow[b]{2}{*}{ Categorias } & \multirow[b]{2}{*}{$\begin{array}{c}\text { Total } \\
n=484 \\
(\%)\end{array}$} & \multicolumn{2}{|c|}{ Causa da orfandade } & \multirow[b]{2}{*}{$p$} \\
\hline & & & $\begin{array}{c}\text { aids } \\
n=269 \\
(\%)\end{array}$ & $\begin{array}{c}\text { Homicídio } \\
n=215 \\
(\%)\end{array}$ & \\
\hline \multirow[t]{3}{*}{ Estado nutricional } & Baixa estatura & 2.2 & 3.1 & 1.8 & 0.52 \\
\hline & Baixo peso & 1.6 & 2.0 & 1.5 & 0.74 \\
\hline & Excesso de peso & 19.5 & 22.0 & 18.6 & 0.30 \\
\hline Estado de saúde atual & Regular / ruim & 15.4 & 15.9 & 15.2 & 0.85 \\
\hline Internação no ano anterior à entrevista & Sim & 4.4 & 4.8 & 4.3 & 0.81 \\
\hline
\end{tabular}

coeficiente de determinação do modelo foi baixo e correspondeu a $3,5 \%$.

No bloco distal da altura, observou-se que o índice nutricional associou-se diretamente ao escore econômico e inversamente à idade do órfão. No bloco intermediário, observou-se que a altura associou-se ao tipo de orfandade, causa da orfandade e situação econômica após o óbito ao se ajustar para as demais variáveis do bloco. $\mathrm{O}$ modelo múltiplo final indicou que a altura associou-se ao escore econômico e à idade do órfão, ajustados pelas demais variáveis intermediárias selecionadas (Tabela 4). O coeficiente de determinação correspondeu a $11,25 \%$. 
Tabela 4 - Efeitos dos determinantes do estado nutricional sobre os índices antropométricos em crianças órfãs por aids ou homicídio, de 5 a 14 anos de idade. São Paulo, Brasil, 2006-07.

Table 4 - Effects of nutritional status determinants on anthropometric indexes in children orphaned by aids or by homicide aged 5-14 years. São Paulo, Brazil, 2006-07.

\begin{tabular}{|c|c|c|c|c|c|}
\hline Determinantes & Categorias & Coeficiente b & $p$ & $\begin{array}{c}\text { Coeficiente b } \\
\text { ajustado }\end{array}$ & $\mathrm{p}$ \\
\hline \multicolumn{6}{|l|}{ Z-score IMC-para-idade } \\
\hline \multicolumn{6}{|l|}{ Determinantes básicos } \\
\hline Bloco 1a & Salário mínimo per capita & & & & \\
\hline \multirow[t]{5}{*}{ Variáveis socioeconômicas } & $0 \mid--1 / 4$ (baseline) & & & & \\
\hline & $1 / 4 \mid--1 / 2$ & -0.11 & 0.55 & -0.14 & 0.41 \\
\hline & $1 / 2 \mid--1$ & 0.17 & 0.33 & 0.14 & 0.40 \\
\hline & $1 \mid-2$ & -0.12 & 0.61 & -0.09 & 0.70 \\
\hline & $>=2$ & 0.73 & 0.02 & 0.70 & 0.03 \\
\hline \multicolumn{6}{|l|}{ Bloco 1b } \\
\hline \multirow[t]{4}{*}{ Variáveis biológicas } & Idade do órfão & -0.02 & 0.30 & -0.02 & 0.42 \\
\hline & Sexo do órfão & & & & \\
\hline & Masculino (baseline) & & & & \\
\hline & Feminino & -0.05 & 0.65 & -0.05 & 0.61 \\
\hline \multicolumn{6}{|c|}{ Determ. intermediários } \\
\hline Bloco 2 & Tipo da orfandade & & & & \\
\hline Variáveis relacionadas & Ambos os pais (baseline) & & & & \\
\hline \multirow[t]{2}{*}{ à orfandade } & Paterna & 0.27 & 0.09 & 0.15 & 0.36 \\
\hline & Materna & -0.16 & 0.38 & -0.19 & 0.33 \\
\hline \multicolumn{6}{|l|}{ Z-Score altura-para-idade } \\
\hline \multicolumn{6}{|l|}{ Determinantes básicos } \\
\hline Bloco 1a & Escore econômico & & & & \\
\hline \multirow[t]{3}{*}{ Var. socioeconômicas } & $11^{\circ}$ terço (baseline) & & & & \\
\hline & $2^{\circ}$ terço & 0.53 & 0.00 & 0.50 & 0.01 \\
\hline & $3^{\circ}$ terço & 0.46 & 0.01 & 0.45 & 0.01 \\
\hline \multicolumn{6}{|l|}{ Bloco 1b } \\
\hline \multirow[t]{4}{*}{ Variáveis biológicas } & Idade do órfão & -0.08 & 0.00 & -0.08 & 0.00 \\
\hline & Sexo do órfão & & & & \\
\hline & Masculino (baseline) & & & & \\
\hline & Feminino & -0.03 & 0.76 & 0.00 & 0.99 \\
\hline \multicolumn{6}{|c|}{ Determ. intermediários } \\
\hline Bloco 2 & Tipo da orfandade & & & & \\
\hline Variáveis relacionadas & Ambos os pais (baseline) & & & & \\
\hline \multirow[t]{9}{*}{ à orfandade } & Paterna & -0.43 & 0.15 & -0.47 & 0.06 \\
\hline & Materna & -0.45 & 0.09 & -0.41 & 0.07 \\
\hline & Cauda da orfandade & & & & \\
\hline & aids (baseline) & & & & \\
\hline & Homicídios & 0.26 & 0.11 & 0.14 & 0.35 \\
\hline & Situação econômica após o óbito & & & & \\
\hline & Melhorou (baseline) & & & & \\
\hline & Piorou & -0.21 & 0.34 & -0.24 & 0.20 \\
\hline & Não mudou & -0.35 & 0.11 & -0.16 & 0.40 \\
\hline
\end{tabular}


A declaração sobre a condição econômica após o óbito não mostrou interação com nível econômico (renda familiar per capita ou escore econômico) do órfão.

\section{Discussão}

Tanto quanto se pode investigar na literatura, este é o primeiro estudo com informações sobre o estado nutricional de órfãos brasileiros. Os principais achados mostraram baixas prevalências de déficits de peso e de altura, e elevada prevalência de excesso de peso. Além disso, os índices nutricionais não se mostraram associados a variáveis relacionadas à orfandade.

Identificou-se elevada prevalência de orfandade paterna, principalmente entre órfãos por homicídio. Este grupo e seus cuidadores apresentaram idade mais nova que os órfãos por aids. Além disso, a maioria dos cuidadores era do sexo feminino, assim como estudos similares com órfãos por aids de outros países ${ }^{10,23}$.

As diferenças detectadas entre os grupos de crianças poderiam, inicialmente, sugerir maior vulnerabilidade nutricional entre órfãos por homicídios por serem mais novas, terem perdido principalmente o pai, por apresentarem a maior frequência de relato sobre a piora da condição econômica após o óbito e pelo maior tempo da orfandade. Contudo, as análises dos indicadores nutricionais utilizados não apontaram para esta direção.

A prevalência dos indicadores nutricionais não diferiu entre os órfãos por aids e homicídio em São Paulo e se mostrou semelhante ao perfil identificado entre crianças e adolescentes brasileiros que viviam em condições urbanas semelhantes ${ }^{24}$. Quando comparados aos outros países, notou-se que a prevalência de déficits nutricionais (peso ou altura) entre órfãos paulistanos foi ainda menor, como foi identificado em estudo similar com órfãos por aids de 6 a 12 anos de idade da Tailândia ${ }^{23}$. Estudos conduzidos na África também mostraram maior prevalência de déficits: a frequência de baixo peso entre órfãos variou de $13 \%$ em Kampala ${ }^{10} \mathrm{a}$
21\% no Quênia ${ }^{25}$.É importante destacar que estes resultados se restringiram às crianças africanas abaixo de cinco anos de idade.

No período ao qual correspondeu a infância dos órfãos deste estudo, a cidade de São Paulo caracterizou-se por apresentar ambiente de menor risco ao crescimento das crianças. Os principais determinantes da redução dos déficits nutricionais em crianças foram o aumento do poder aquisitivo das famílias, da escolaridade das mães, melhoria das condições de moradia, saneamento do meio, maior acesso aos serviços básicos de saúde e antecedentes reprodutivos adequados ${ }^{26}$. As mudanças positivas no cenário brasileiro persistiram e, recentemente, a prevalência de baixa estatura é praticamente ausente no país ${ }^{27}$. Essas condições criaram rede de proteção pública que reduziu a exposição das crianças aos déficits nutricionais, que provavelmente alcançaram até mesmo grupos de crianças vulneráveis aos agravos de saúde como órfãos de São Paulo.

Outros aspectos importantes observados foram que, em média, as crianças ficaram órfãs em idade maior que dois anos, época posterior a um dos períodos mais críticos do crescimento humano, e não houve associação entre a alteração da condição econômica após o óbito e os valores $\mathrm{Z}$ de altura, nem mesmo após o teste de interação com o nível econômico da criança. Os resultados sugeriram que, embora grande parte dos cuidadores tenha relatado piora da condição econômica após o óbito, especialmente entre órfãos por homicídios, esta não foi de magnitude que chegasse a afetar o crescimento infantil. O escore econômico associou-se à altura da criança, independentemente das condições relacionadas à orfandade. Segundo Rivers et al. ${ }^{8}$, o impacto negativo deste aspecto advindo da orfandade pode ter sido amenizado por vantagens nutricionais adquiridas no útero ou no início da vida da criança.

O estado nutricional de órfãos de São Paulo foi semelhante ao observado em pesquisa representativa com crianças e adolescentes brasileiros ${ }^{24}$. A elevada 
prevalência de excesso de peso sugere que parte dos fenômenos identificados no presente estudo está mais alinhada à transição nutricional do que às condições adquiridas com a orfandade. De modo geral, a transição nutricional se caracteriza pela modificação do perfil nutricional de uma população decorrente das mudanças do padrão dietético e de atividade física. Esta mudança é determinada basicamente pelas mudanças econômicas, demográficas, ambientais e culturais em uma sociedade, e tem ocorrido principalmente entre os mais pobres ${ }^{28}$.

Além disso, a análise hierárquica mostrou ausência de efeito dos aspectos relacionados à orfandade e que, tanto para o IMC quanto para a altura, o principal determinante foi de natureza econômica.

A principal limitação deste estudo foi perda amostral devida ao desconhecimento do paradeiro dos órfãos, a qual pôde ser parcialmente corrigida com uso de pesos e ajuste para a não resposta. A alta mobilidade espacial de populações pobres e afetadas pelas mortes por aids e homicídio, em especial em grandes metrópoles como São Paulo, explica o fato de os órfãos poderem ser considerados um grupo de difícil acesso.

\section{Conclusão}

Os resultados do presente estudo sugerem que os órfãos por aids ou homicídio do município de São Paulo não possuem riscos adicionais para o seu estado nutricional. O perfil identificado no presente estudo é compatível com a transição nutricional vivenciada pelas demais crianças e adolescentes brasileiros. A prevenção da obesidade como objetivo das políticas de alimentação e nutrição deve se estender também a grupos vulneráveis como o de órfãos do presente estudo.

\section{Referências}

1. Ainsworth M, Semali I. The Impact of Adult Deaths on Children's Health in North Somalia. Washington (DC): World Bank; 2003.

2. Sharma M. Orphans in Malawi: prevalence, outcomes, and targeting of services. International Food Policy Research Institute. Washington (DC): World Bank, 2005.

3. UNICEF - United Nations Children's Fund. Situação Mundial da Infância. Report; 2005. Disponível em http://www.UNICEF.org. [Acessado em 15 de outubro de 2006]

4. Kikafunda JK, Namusoke HK. Nutritional status of HIV/aids orphaned children in households headed by the elderly in Rakai District, South Western, Uganda. AJFAND 2006; 6: 1-18.

5. Lindblade KA, Odhiambo F, Rosen DH, DeCock KM (2003) Health and nutritional status of orphans $<6$ years old cared for by relatives in western Kenya. Trop Med Int Health 2003; 8: 67-72.

6. He Z, Ji C. Nutritional status, psychological well-being and the quality of life of aids orphans in rural Henan Province, China. Trop Med Int Health 2007; 12: 1180-90.

7. Siaens C, Subbarao K, Wodon Q. Are orphans especially vulnerable? Evidence from Rwanda. Washington (DC): World Bank; 2003.
8. Rivers J, Mason J, Silvestre E, Gillespie S, Mahy M, Monasch R. Impact of orphanhood on underweight prevalence in sub-Saharan Africa. Food Nutr Bull 2006; 29: $32-42$.

9. UNAids - The United Nations Joint Programme on HIV/ aids, USaids - The United States Agency for International Development's, UNICEF - United Nations Children's Fund. Children on the brick - A joint report of new orphan estimates and a framework for action. UNICEF report. New York; 2004.

10. Sarker M, Neckermann C, Muller O. Assessing the health status of young aids and other orphans in Kampala, Uganda. Trop Med Int Health 2005; 10: 210-5.

11. Watts H, Gregson S, Saito S, Lopman B, Beasley M, Monasch R. Poorer health and nutritional outcomes in orphans and vulnerable young children not explained by greater exposure to extreme poverty in Zimbabwe. Trop Med Int Health 2007, 12: 584-93.

12. Ministério da Saúde. Fundação Nacional da Saúde. Centro Nacional de Epidemiologia. Sistema de Informação sobre Mortalidade - SIM. Disponível em http://tabnet.datasus.gov.br [Acessado em 6 de agosto de 2007]

13. Doring M, França-Junior I, Stella I. Factors associated with institutionalization of orphans due to aids in a population-based survey in Porto Alegre, Brazil. Aids 2005; 19(S4): 59-63 
14. Barros CRSB, Zucchi EM, França-Junior I. Nível de atividade física de crianças e adolescentes órfãos por aids. Rev Bras Epidemiol 2010; 13(3): 446-56.

15. Lohman TG, Roche AF, Martorell R. Anthropometric standardization reference manual. Champaign, IL: Human Kinetics Books; 1988.

16. Conde WL \& Monteiro CA. Body mass index cutoff points for evaluation of nutritional status in Brazilian children and adolescents. J Pediatr 2006; 82: 266-72.

17. World Health Organization. Expert Committee on Physical Status: the use and interpretation of anthropometry. Report. Geneva; 1995. (WHO- Technical Report Series 854).

18. Cole TJ. A critique of the NCHS weight for height standard. Hum Biol 1985; 57: 183-96.

19. Cole TJ, Flegal KM, Nicholls D, Jackson AA. Body mass index cut offs to define thinness in children and adolescents: international survey. BMJ 2007; 335: 194.

20. De Onis M, Onyango AW, Borghi E, Siaym A, Nishida C, Siekmann J Development of a WHO growth reference for school-aged children and adolescents. Bull World Health Organ 2007; 85: 660-7.

21. United Nations Children's Fund. Strategy for improved nutrition of children and women in developing countries. UNICEF report. New York; 1990.
22. Norgan NG. Body mass index and body energy stores in developing countries. Eur J Clin Nutr 1990; 44: 79-84.

23. Isaranurug S, Chompikul J. Emotional Development and Nutritional Status of HIV/aids Orphaned Children Aged 6-12 Years Old in Thailand. Matern Child Health J 2009; 13: $138-143$

24. Instituto Brasileiro de Geografia e Estatística. Pesquisa de Orçamentos Familiares 2002-2003 (POF). Antropometria e Estado Nutricional de crianças e adolescentes brasileiros. Rio de Janeiro: IBGE; 2006.

25. Mishra V, Arnold F, Otieno F, Cross A, Hong R. Education and nutritional status of orphans and children of HIVinfected parents in Kenya. Aids Educ Prev 2007; 19(5): 383-95.

26. Monteiro CA, Conde WL. Tendência secular do crescimento pós-natal na cidade de São Paulo (19741996). Rev Saúde Publica 2000; 34: S41-S51.

27. Monteiro CA, Benicio MHA, Konno SC, Silva ACF, Lima ALL, Conde WL. Causes for the decline in child undernutrition in Brazil, 1996-2007. Rev Saúde Pública 2009; 43: 1-8.

28. Popkin BM. The shift in stages of the nutrition transition in the developing world differs from past experiences! Mal J Nutr 2002; 8(1): 109-24.

Recebido em: 11/05/11 Versão final apresentada em: 14/05/12 Aprovado em: 17/05/12 